\author{
C. BORCHERS ${ }^{1, \infty}$ \\ T. SCHMIDT ${ }^{2}$ \\ F. GÄRTNER ${ }^{2}$ \\ H. KREYE ${ }^{2}$
}

\title{
High strain rate deformation microstructures of stainless steel 316L by cold spraying and explosive powder compaction
}

\footnotetext{
${ }^{1}$ Institute for Materials Physics, University of Göttingen, Friedrich-Hund-Platz 1, 37077 Göttingen, Germany

${ }^{2}$ Institute for Materials Technology, Helmut-Schmidt-University of the Federal Armed Forces, 22043 Hamburg, Germany
}

\begin{abstract}
Received: 5 June 2007/Accepted: 21 September 2007
Published online: 2 November 2007 • @ Springer-Verlag 2007

ABSTRACT Cold spraying is a new coating technique in which dense, tightly bonded coatings form only due to the high kinetic energy of impinging particles of the spray powder. These particles are still in the solid state during impact. Explosive powder compaction is a technique where powder is consolidated by a shock wave. In the shock front the powder is deformed under high strain rates, which under suitable conditions results in high-strength bonding of the particles. Thus, the microstructural features obtained by both techniques should be similar. This study correlates the microstructure of cold-sprayed 316L austenitic steel coatings in comparison to the microstructure of 316L samples obtained by explosive compaction. The results provide insight into the prevailing local deformation mechanisms, as well as into the physical background of observed phase transformations.
\end{abstract}

PACS 81.15.Rs; 61.72.Ff; 62.50.+p; 64.60.My; 64.70.Kb

\section{$1 \quad$ Introduction}

In conventional thermal spray techniques like flame spraying, high-velocity oxy-fuel spraying or plasma spraying, the spray process is accompanied by a complete or partial melting of the metallic powder feedstock material, which can result in high residual stresses in the coatings due to the shrinkage during rapid solidification on the substrate, and also in oxidisation of the material, which takes place in ambient atmosphere. The former can cause delamination of the coating, whereas the latter has direct influence on phase distribution and composition because of the formation of oxides at particle-particle interfaces which determine physical and mechanical properties of the coating, e.g. its resistivity or its strength.

In the new coating technique of cold spraying (CS), bonding of particles is a result of extensive plastic deformation and related phenomena at the interface [1-8]. In this process, solid particles in size ranges between typically 5 and $50 \mu \mathrm{m}$ are introduced into the high-pressure chamber of a convergingdiverging de Laval type nozzle and are accelerated in a supersonic stream of inert gas. The particles reach velocities $v_{\mathrm{CS}}$

Fax: +49-551-39-5012, E-mail: chris@ump.gwdg.de ranging from 400 to $1200 \mathrm{~m} / \mathrm{s}$ and build up dense coatings only due to their kinetic energy upon impact. Because of the low thermal influence, the method can open up new applications for oxidation-sensitive metastable or nanocrystalline materials. Whether particles stick to the substrate and coating or bounce back is related to the degree and rate of plastic deformation at the substrate-particle and particle-particle interfaces. Since such a severe transition is correlated with massive changes in local microstructures, microscopic methods are applied to investigate the internal interfaces between particles in cold-sprayed $316 \mathrm{~L}$ austenitic stainless steel coatings. The principle of CS coating-buildup is sketched in Fig. 1a.

In explosive powder compaction (EPC), a powder is loaded by a strong shock wave. Because of extremely rapid compression of the powder, dense solids of metals and ceramics can be produced by an appropriate selection of shock pressure and duration [9-14]. Particle deformation by the shock wave can be considered as an inverse impact of the compressed and accelerated particles behind the shock front towards the non-compressed and non-moving particles in front of the shock front, as sketched in Fig. 1b. Therefore, the bonding conditions are expected to be similar to those obtained in cold spraying, the latter consisting of conventional impacts of more or less single particles, as sketched in Fig. 1a [14]. The strain rate of the deformation and the deformation mechanism strongly depend on the local shock pressure. Because of radial energy input in the cylindrical sample, shock loading in explosive powder compaction can cover a wide pressure range in a single experiment, until the velocity of the shock wave focussed towards the centre reaches the detonation velocity at the Mach zone close to the centre of the sample [14].

In analogy with explosive cladding [15-17], successful bonding in powder compaction and cold spraying has been related to critical conditions for extensive plastic deformation at the particle-particle interface $[8,14,18-20]$. This is accompanied by adiabatic shear instabilities in near-surface regions of the particles resulting in the appearance of fast-travelling jets. To reach the regime of adiabatic shear instabilities, a critical particle velocity $v_{\text {cr }}$ has to be attained, below which no successful bonding is observed $[5,6,18]$. In explosive powder compaction a change in deformation mechanism under such critical conditions is reported [14]. The particle deformation pattern changes from a symmetric angular shape to an asymmetric parachute shape [13,14]. Apart from the material 

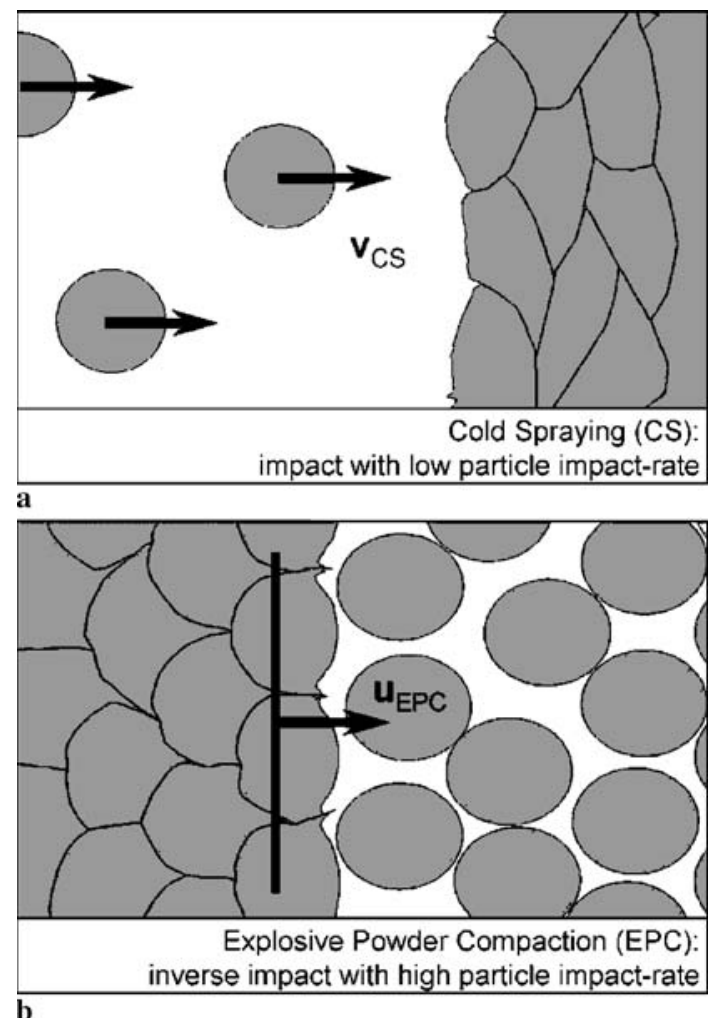

FIGURE 1 Schematic of particle impact and impact velocity $v_{\text {CS }}$ in cold spraying (a), and schematic of inverse impact and material velocity $u_{\mathrm{EPC}}$ in explosive powder compaction (b)

strength, density, melting temperature, and heat capacity, $v_{\mathrm{cr}}$ is also dependent on the particle impact temperature and the particle size [19]. Larger and hotter particles require a lower velocity for a high-strength bonding and dense coatings.

It is the aim of this work to compare the deformation behaviour of 316L stainless steel powders in cold spraying and explosive powder compaction and to correlate observed microstructures with the respective energy inputs. The obtained results should provide a deeper physical understanding of the high strain rate deformation mechanisms. In addition, the results should be useful to predict the behaviour of engineering materials subjected to high strain rate deformation.

\section{2}

\section{Experimental}

For the experiments three different powder size distributions, $<22 \mu \mathrm{m}$ (fine), 15 to $45 \mu \mathrm{m}$ (medium) and 53 to $177 \mu \mathrm{m}$ (coarse) of a gas atomised austenitic steel 316L powder from Carpenter have been used. The common notation $-45+15 \mu \mathrm{m}$ for the powder size 15 to $45 \mu \mathrm{m}$ and analogous notation for the other size cuts will be used. Figure 2 shows scanning electron microscopy (SEM) micrographs of the etched particle cross sections of the three powder size cuts. It can be seen that the particles are multi-crystalline with grain sizes in the $\mu \mathrm{m}$ range. The composition of the steel $316 \mathrm{~L}$ base material used for powder production is listed in Table 1. The oxygen and the nitrogen contents as well as the hardness of the different-size fractions are listed in Table 2. Oxygen and nitrogen contents are increasing with decreasing particle size, because the higher surface to volume ratio of the finer size frac-
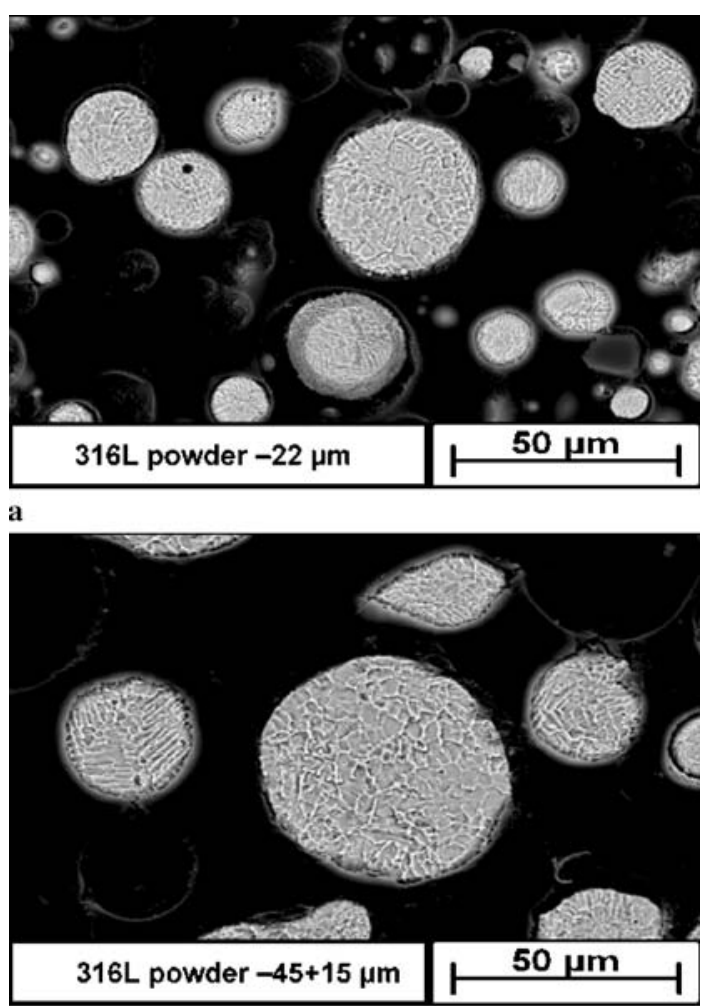

b

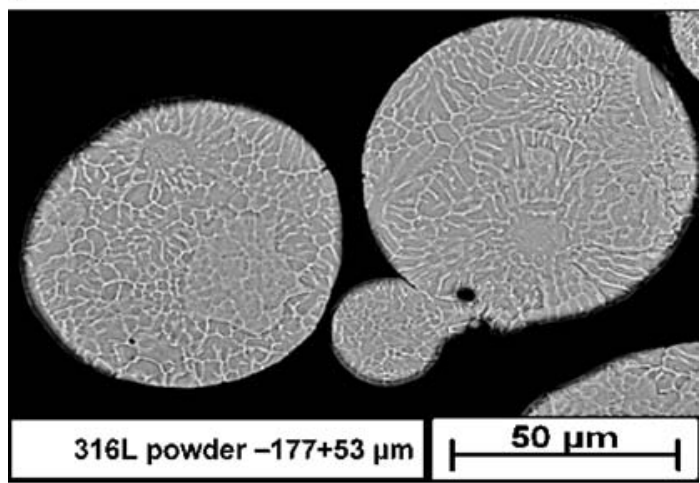

c

FIGURE 2 SEM micrographs of cross sections of 316L feedstock powders of different-size cuts: (a) $-22 \mu \mathrm{m}$ (fine), (b) $-45+15 \mu \mathrm{m}$ (medium), and (c) $-177+53 \mu \mathrm{m}$ (coarse)

\begin{tabular}{lcccccccc}
\hline $\mathrm{C}$ & $\mathrm{Cr}$ & $\mathrm{Ni}$ & $\mathrm{Mo}$ & $\mathrm{Mn}$ & $\mathrm{Si}$ & $\mathrm{P}$ & $\mathrm{S}$ & $\mathrm{Fe}$ \\
\hline 0.017 & 16.3 & 10.8 & 2.11 & 1.41 & 0.43 & 0.025 & 0.004 & Balance
\end{tabular}

TABLE 1 Chemical composition of the AISI 316L stainless steel powder (wt.\%)

tions makes them more sensible to contaminations. But, this obviously does not significantly influence the hardness of the particles.

The critical velocities $v_{\text {cr }}$ for cold spraying listed in Table 3 are calculated for the mean particle diameter of the three size fractions and for two different impact temperatures [14]. The experimental determination of critical velocities is reported in $[7,18,19]$, and they correspond well with the calculated values. The results listed in Table 3 demonstrate that with increasing particle size and increasing temperature the crit- 


\begin{tabular}{lccc}
\hline & $\begin{array}{c}-22 \mu \mathrm{m} \\
\text { Fine }\end{array}$ & $\begin{array}{c}-45+15 \mu \mathrm{m} \\
\text { Medium }\end{array}$ & $\begin{array}{c}-177+53 \mu \mathrm{m} \\
\text { Coarse }\end{array}$ \\
\hline Oxygen content/\% & 0.05 & 0.03 & 0.01 \\
Nitrogen content/\% & 0.14 & 0.09 & 0.02 \\
Hardness HV0.01 & 200 & 186 & 185 \\
\hline
\end{tabular}

TABLE 2 Experimentally determined hardness and oxygen and nitrogen contents of different-size cuts of stainless steel $316 \mathrm{~L}$ powder

\begin{tabular}{|c|c|c|c|}
\hline & $\begin{array}{c}-22 \mu \mathrm{m} \\
d_{\mathrm{m}}=15 \mu \mathrm{m}\end{array}$ & $\begin{array}{c}-45+15 \mu \mathrm{m} \\
d_{\mathrm{m}}=30 \mu \mathrm{m}\end{array}$ & $\begin{array}{c}-177+53 \mu \mathrm{m} \\
d_{\mathrm{m}}=115 \mu \mathrm{m}\end{array}$ \\
\hline$v_{\mathrm{cr}} /(\mathrm{m} / \mathrm{s}), \quad T_{\mathrm{i}}=20^{\circ} \mathrm{C}$ & 650 & 590 & 490 \\
\hline$v_{\mathrm{cr}} /(\mathrm{m} / \mathrm{s}), \quad T_{\mathrm{i}}=300^{\circ} \mathrm{C}$ & 590 & 530 & 440 \\
\hline
\end{tabular}

TABLE 3 Critical velocities for cold-sprayed steel 316L powders, calculated for the mean particle diameter $d_{\mathrm{m}}$ of different powder size cuts at different impact temperatures $T_{\mathrm{i}}$ [19]

ical velocity decreases. For the cold-spray experiments, nitrogen at $25 \mathrm{bar}$ and $500^{\circ} \mathrm{C}$ or helium at $30 \mathrm{bar}$ and $400^{\circ} \mathrm{C}$ were used as process gas to accelerate the particles of the fine $(-22 \mu \mathrm{m})$ and the medium $(-45+15 \mu \mathrm{m})$ size cuts, respectively. As carrier gas in both cases nitrogen was used. The impact conditions are calculated in relation to the mean diameter of the powders used. Under nitrogen spray conditions the particles of the fine powder $\left(d_{\mathrm{m}}=15 \mu \mathrm{m}\right)$ impinge to the substrate with $600 \mathrm{~m} / \mathrm{s}$ and $300^{\circ} \mathrm{C}$; compare Fig. $6 \mathrm{a}$ and b. Using helium as process gas impact velocities are significantly higher and the impact temperatures are significantly lower. In this case, the particles of the fine powder impact with $850 \mathrm{~m} / \mathrm{s}$ and $-20^{\circ} \mathrm{C}(253 \mathrm{~K})$ and the particles of the medium powder $\left(d_{\mathrm{m}}=30 \mu \mathrm{m}\right)$ with $800 \mathrm{~m} / \mathrm{s}$ and $100^{\circ} \mathrm{C}$; compare Fig. $6 \mathrm{c}$ and d. More general aspects of the coating technique and also the calculation of impact conditions are reported in $[1-3,5,6,18,19,21]$.

In explosive powder compaction (EPC), the loading conditions are independent of particle size, whereas in cold spraying (CS) they strongly depend on particle size $[14,19]$. Therefore, EPC experiments were performed with $316 \mathrm{~L}$ powder of the fine and the coarse size cuts. Another advantage of EPC
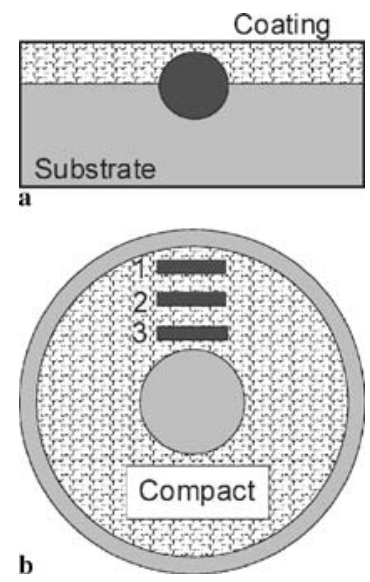

FIGURE 3 Schematic of the sample geometries for TEM. (a) From coldsprayed coatings, side view, and (b) from explosive compacts, top view. Sample 1 is gained from the outside, sample 2 from the middle, and sample 3 from the centre of the compact is that the loading conditions can be varied in a wide range, especially to very high material velocities $u_{\mathrm{EPC}}$. To obtain a wide range of loading conditions, two explosives were used in this work, first ANFO (ammonium nitrate/fuel oil) with a detonation velocity of $3100 \mathrm{~m} / \mathrm{s}$ and a detonation pressure of $2.5 \mathrm{GPa}$ and second an RDX (hexogene)-based explosive with a detonation velocity of $5500 \mathrm{~m} / \mathrm{s}$ and a detonation pressure of 6.8 GPa. In the so-called direct-compaction method (d.c.), the powder is filled into a cylindrical steel container with a homogeneous mandrel in the centre. The ignition point is positioned at the axis of symmetry of the charge. The detonation wave grazes the powder container in the axial direction and sends a radial shock wave into the powder. Due to the radial energy input, the shock pressure increases towards the centre [9]. At the investigated areas, the powder is accelerated to velocities between $500 \mathrm{~m} / \mathrm{s}$ at the outside of the cylindrical ingot and $800 \mathrm{~m} / \mathrm{s}$ at the centre. In the very narrow Mach zone close to the mandrel in the centre of the sample, material velocities higher than $1000 \mathrm{~m} / \mathrm{s}$ cause partial melting or total melting of the $316 \mathrm{~L}$ powder. For the investigation of highly loaded material, additional experiments were performed in the socalled flyer tube (f.t.) configuration. In this method, a copper tube, sheathing the steel tube (powder container) with a radial distance, is used to generate an additional focussing effect to increase the shock pressure in the powder [12]. With this method, a more homogeneous distribution of energy over the sample radius can be obtained. This advantage allowed the production of a comparatively wide zone of material, which was exposed to material velocities higher than $1000 \mathrm{~m} / \mathrm{s}$.

Since in explosive powder compaction the density of the powder influences the deformation of a single particle and prescribes a highly different number of impact events per time unit and area, the material velocities $u_{\text {EPC }}$ in EPC do not correspond exactly to the particle impact velocities $v_{\mathrm{CS}}$ in CS; see Fig. 1. The impact rate generated by the apparently dense powder in front of the fast-travelling shock wave in explosive powder compaction is around $10^{4}$ times higher than the impact rate of more or less single particles in cold spraying, which is 0.01 to $0.1 \mathrm{~g} /\left(\mathrm{mm}^{2} \mathrm{~s}\right)$. As a major difference in comparison to the impact conditions in CS, the high impact rate in EPC generates a significant solid-state compression of the moving material behind the shock front. However, it is possible to give corresponding velocities $[14,19]$, which are given in Table 4. Detailed information on explosive powder compaction can be found in $[9-12,14]$.

X-ray diffraction (XRD) was performed with a X 3000 from SEIFERT (Germany) using Co $K_{\alpha}$ radiation at a preset voltage of $40 \mathrm{kV}$ and a secondary monochromator. Prior to XRD analyses, the surface of cold-sprayed samples was ground and polished to reveal structures from the inside of

\begin{tabular}{lcccc}
\hline & Outside & Middle & Center & Close to mandrel \\
\hline EPC powder velocity & $\sim 500$ & $\sim 600$ & $\sim 800$ & $>1000$ \\
$u_{\mathrm{EPC}} /(\mathrm{m} / \mathrm{s})$ & & & & \\
$\mathrm{CS}$ impact velocity & $\sim 600$ & $\sim 800$ & $\sim 1050$ & $>1400$ \\
$v_{\mathrm{CS}} /(\mathrm{m} / \mathrm{s})$ & & & & \\
\hline
\end{tabular}

TABLE 4 Calculated EPC material velocity $u_{\text {EPC }}$ in EPC and corresponding CS impact velocity $v_{\mathrm{CS}}[14,19]$ 
the coatings. The same procedure was used to investigate cross sections along the axis of EPC samples. By using a tubular aperture, structures at different radii could be distinguished. More detailed microstructural investigations were performed by optical microscopy (OM), scanning electron microscopy (SEM), and transmission electron microscopy (TEM). Scanning electron microscopy was performed with a Philips XL 40. For SEM, cross sections of the coatings on the substrates were prepared and etched to reveal grain boundaries and particle-particle interfaces. To investigate the cold-sprayed coatings by TEM, 3-mm-diameter discs were punched from cross sections, see Fig. 3a. These were polished, dimpled, and ion milled, so that the coating-substrate interface is in the electron-transparent region. This allows us to determine the impact direction. To examine explosively compacted material (EPC) corresponding to different impact energies, sections were cut from different planes on the radius of the samples. Transmission electron microscopy was performed with a Philips EM 420 ST at an acceleration voltage of $120 \mathrm{kV}$. The different geometries to prepare TEM samples from CS coatings and EPC specimens are sketched in Fig. 3. Such samples were also used for XRD analyses.

\section{$3 \quad$ Results \\ 3.1 $\quad X R D$}

Figure 4a shows X-ray diffraction spectra of $316 \mathrm{~L}$ steel powders of different sizes: $-22 \mu \mathrm{m}$ (fine), $-45+15 \mu \mathrm{m}$ (medium), and $-177+53 \mu \mathrm{m}$ (coarse). It is remarkable that the fine powder exhibits $b c c$ besides $f c c$ peaks revealing 10 to $20 \%$ of $b c c$ phase in this powder, while the medium and coarse powders show only $f c c$ peaks. Figure $4 \mathrm{~b}$ shows results from cold-sprayed steel $316 \mathrm{~L}$ coatings, using $\mathrm{N}_{2}$ and $\mathrm{He}$ as process gases, and fine $(-22 \mu \mathrm{m})$ and medium $(-45+15 \mu \mathrm{m})$ powder size cuts. The coatings exhibit only $f c c$ structures. In Fig. 4c, $\mathrm{X}$-ray diffraction spectra of 316L steel explosive compacts are shown. These spectra are taken completely analogously to the scheme shown in Fig. 3 from the outside $\left(u_{\mathrm{EPC}} \approx 500 \mathrm{~m} / \mathrm{s}\right.$, line 1), the middle $\left(u_{\mathrm{EPC}} \approx 600 \mathrm{~m} / \mathrm{s}\right.$, line 2$)$, and the centre $\left(u_{\mathrm{EPC}} \approx 800 \mathrm{~m} / \mathrm{s}\right.$, line 3$)$ of the cylindrical d.c. compact and from a region of a f.t. compact where the material velocity $u_{\text {EPC }}$ was higher than $1000 \mathrm{~m} / \mathrm{s}$, line 4 . In these cases the coarse powder was used. The microstructures are analogous to Fig. 5a-d; see Sect. 3.2. Additionally, line 5 shows a spectrum of a sample from the middle region of a d.c. compact $\left(u_{\mathrm{EPC}} \approx 600 \mathrm{~m} / \mathrm{s}\right)$, where the fine powder $(-22 \mu \mathrm{m})$ was used. It can clearly be seen that the compacts consist mainly of pure $f c c$ material, which is also the case for the d.c. compact of the fine powder. In the case of the f.t. compact $\left(u_{\mathrm{EPC}}>1000 \mathrm{~m} / \mathrm{s}\right)$ generated with coarse powder, a slight amount of $b c c$ can be seen.

\section{$3.2 \quad O M / S E M$}

The optical micrographs in Fig. 5 show the structure of the d.c. and the f.t. compacted coarse $(-177+$ $53 \mu \mathrm{m})$ powder at different loading conditions and positions (compare Fig. $3 \mathrm{~b}$ ): (a) outside, $u_{\mathrm{EPC}} \approx 500 \mathrm{~m} / \mathrm{s}$, corresponding to TEM sample 1 . The particles are comparatively weakly deformed and are just above the critical conditions, where bonding by shear instabilities starts to occur and the transition of the deformation pattern from a symmetric angular shape to the asymmetric parachute shape takes place $[13,14]$. This typical parachute morphology with extensive plastic flow at some regions of the interface, shown by highly elongated grains and welded areas, can be observed in the presented microstructures. In the middle region (b), $u_{\mathrm{EPC}} \approx 600 \mathrm{~m} / \mathrm{s}$, corresponding to TEM sample 2 , the particles are strongly deformed, most particle-particle inter-

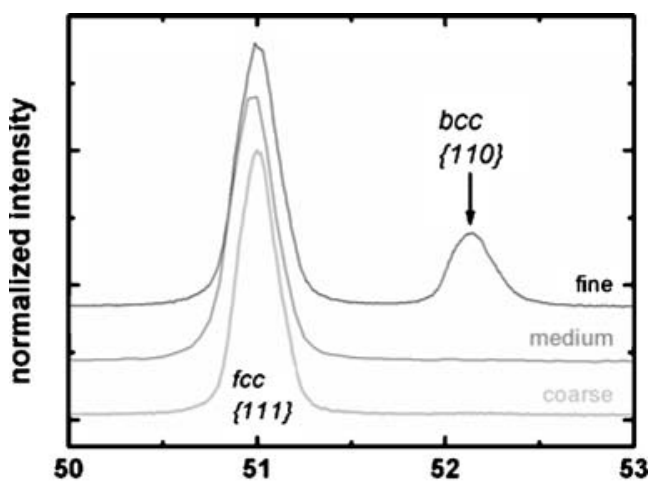

a
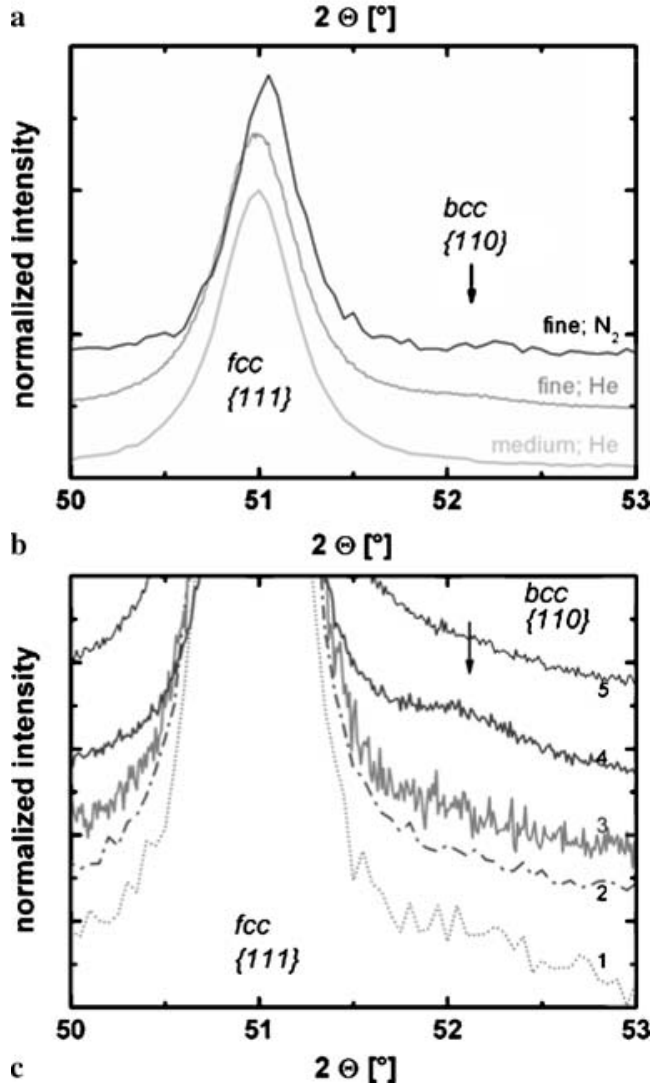

FIGURE 4 X-ray diffraction patterns of (a) feedstock powder, (b) coldsprayed coatings using $\mathrm{N}_{2}$ and $\mathrm{He}$ as process gas, and (c) explosively compacted samples. The notations fine, medium, and coarse are used for powder sizes of $-22 \mu \mathrm{m},-45+15 \mu \mathrm{m}$, and $-177+53 \mu \mathrm{m}$, respectively. The spectra were collected from compacts, produced using the direct-compaction (d.c.) method, taken from the outside, the middle, and the centre of the compacts, corresponding to samples 1, 2, and 3, respectively, in (c) and in Fig. 3. Line 4 in (c) is the spectrum of coarse powder compacted with the flyer tube compaction method, and line 5 the spectrum of fine powder compacted in d.c. mode. Line 4 exhibits a small $b c c\{110\}$ peak 


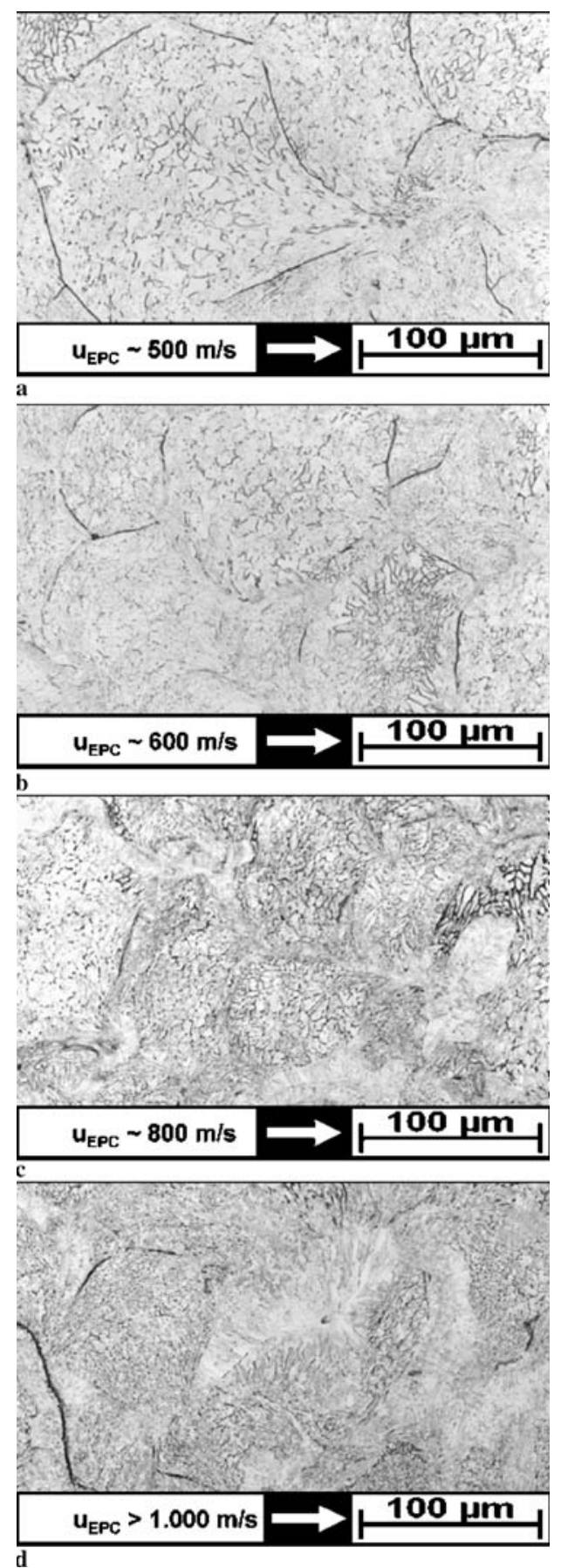

FIGURE 5 OM of typical microstructures of explosively compacted (d.c.) steel 316L powders (powder size: $-175+53 \mu \mathrm{m}$ ) cut from different positions on the radius of the cylindrical d.c. compact. (a) Outside, (b) middle, and (c) centre. (a), (b), and (c) correspond to samples 1, 2, and 3 in Fig. 3, respectively. Micrograph (d) is taken from a compact produced using the flyer tube method. The arrows indicate the moving direction of the consolidated material front

faces are welded and partially show heat-influenced zones of up to $10 \mu \mathrm{m}$ from the interface into the particle. In the centre region $(\mathrm{c}), u_{\mathrm{EPC}} \approx 800 \mathrm{~m} / \mathrm{s}$, the particle-particle interfaces are strongly welded and it is difficult to identify the individual particles. The content of heat-influenced zones increases. Molten and solidified regions of $20 \mu \mathrm{m}$ in diameter can be detected. Figure 5d finally shows a microstructure of

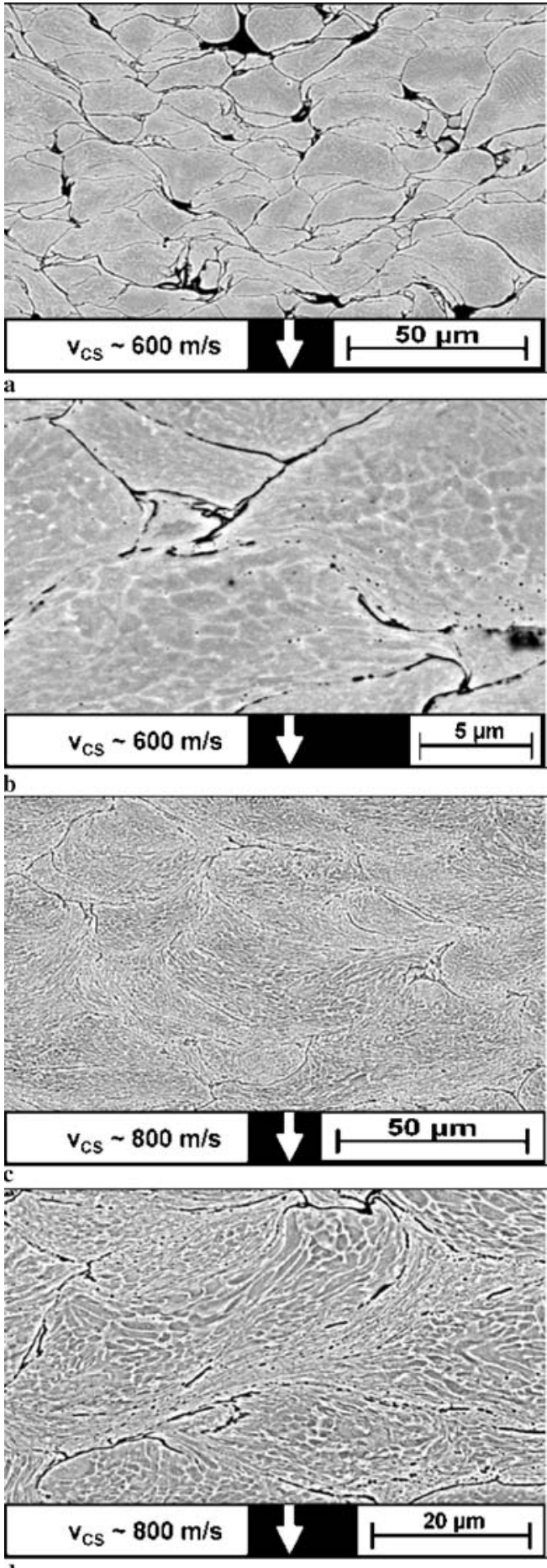

FIGURE 6 SEM micrographs of (a) cold-sprayed steel 316L coating using $\mathrm{N}_{2}$ as process gas $\left(v_{\mathrm{CS}} \approx 600 \mathrm{~m} / \mathrm{s}\right.$, powder size: $\left.<22 \mu \mathrm{m}\right),($ b) the same coating in a higher magnification, (c) cold-sprayed steel $316 \mathrm{~L}$ coating using $\mathrm{He}$ as process gas $\left(v_{\mathrm{CS}} \approx 800 \mathrm{~m} / \mathrm{s}\right.$, powder size: $\left.-45+15 \mu \mathrm{m}\right),(\mathbf{d})$ the same coating in a higher magnification. The arrows indicate the particle impact direction

a f.t. compact where the material velocity reached a value of more than $1000 \mathrm{~m} / \mathrm{s}$. Here, the solidified regions reach a volume of 10 to $20 \%$ and a extension of up to $50 \mu \mathrm{m}$. The shrinkage of the material during solidification causes some pores. 


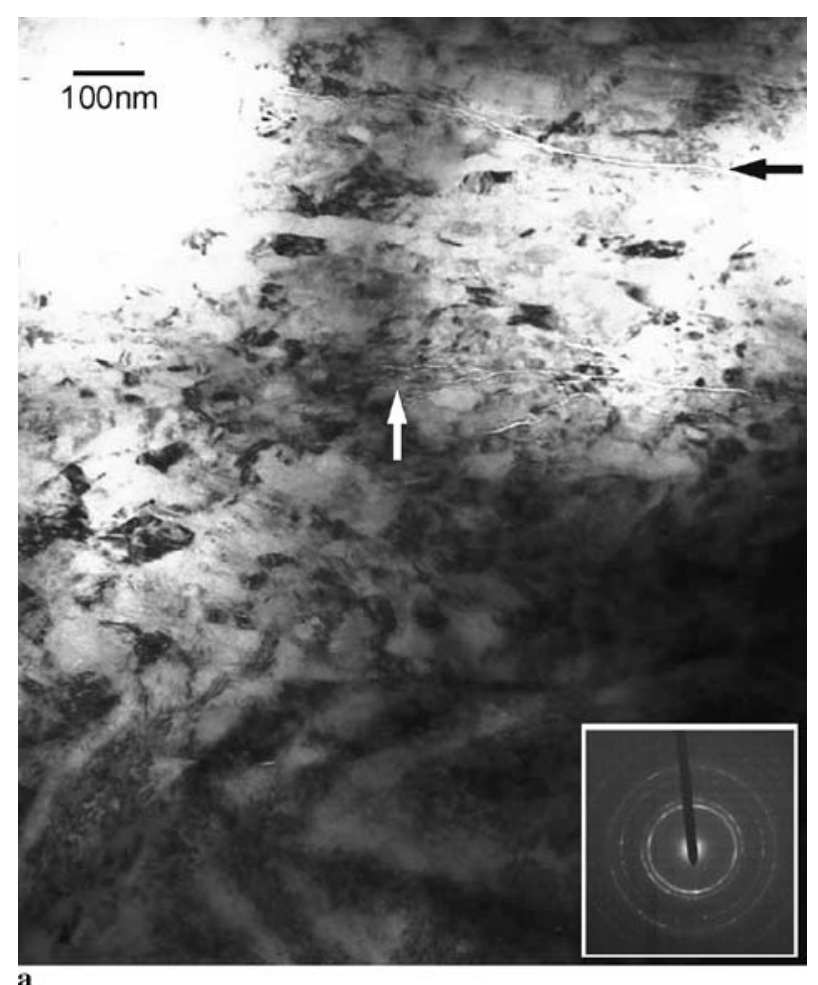

a

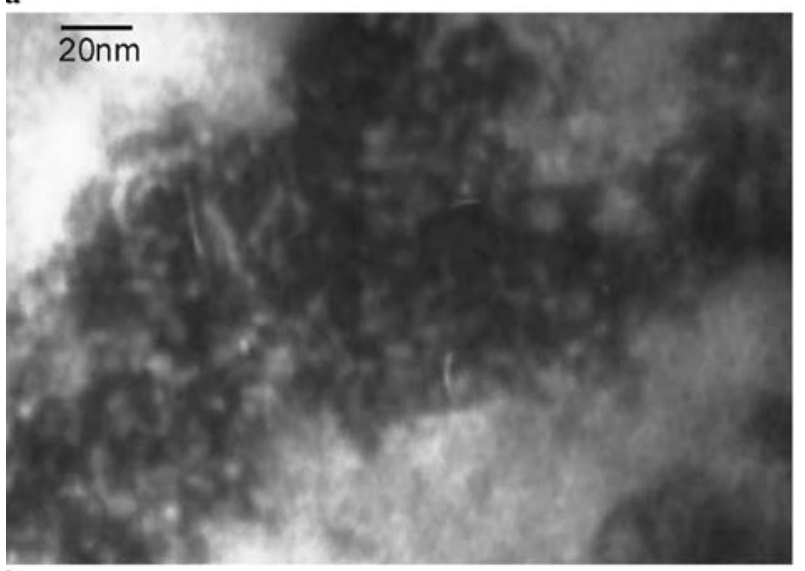

b

FIGURE 7 (a) TEM micrograph of a cold-sprayed steel 316L powder using $\mathrm{N}_{2}$ as process gas (powder size: $<22 \mu \mathrm{m}$ ). A particle-particle boundary is marked with a black arrow, a boundary with a branched structure is marked with a white arrow. (b) Close-up of a grain exhibiting numerous coffeebean-like contrasts, bright in the micrograph. The inset in (a) shows that the coating is pure $f c c$

The SEM micrographs in Fig. 6 show the etched microstructures of $(\mathrm{a}, \mathrm{b})$ a cold-sprayed steel $316 \mathrm{~L}$ coating produced by using the powder size of $-22 \mu \mathrm{m}$ and $\mathrm{N}_{2}$ as process gas, and (c, d) a cold-sprayed steel $316 \mathrm{~L}$ coating using the medium powder size cut of $-45+15 \mu \mathrm{m}$ and $\mathrm{He}$ as process gas. The particle-particle interfaces appear dark; grain boundaries within the particles are bright. In some places, the interparticle bonds are so tight that in these micrographs the interface seems to be disrupted. Especially in the He-sprayed coating, the amount of these areas is quite high and, therefore, a high coating strength can be assumed. The heavy deformation of the particles is evident, since the individual particles are by no means spherical; they are rather flattened. More-
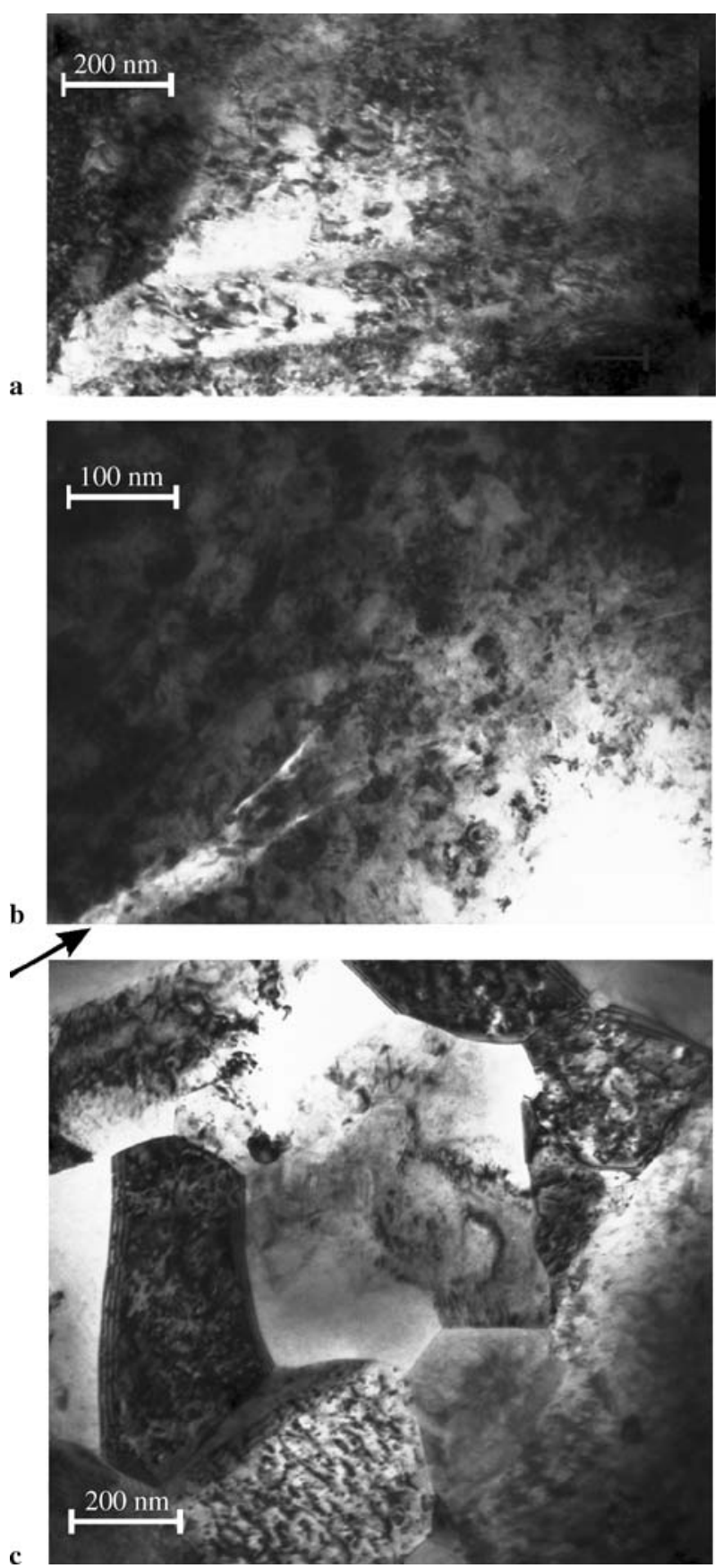

FIGURE 8 TEM micrographs of explosively compacted (d.c.) steel $316 \mathrm{~L}$ powders (powder size: $-175+53 \mu \mathrm{m}$ ); (a) cut from the outside of the radius of the cylindrical compact (sample 1), (b) cut from the middle (sample 2), and (c) cut from the centre (sample 3). In (b), a particle-particle interface is marked with an arrow

over, some individual grains at particle-particle interfaces are highly elongated.

\subsection{TEM}

In the TEM micrograph Fig. 7a, the main features of the microstructure near interparticle interfaces of a coldsprayed 316L austenitic steel coating can be seen. A particleparticle interface is marked with a black arrow. The white spots in the interface are holes which are the result of ion 
milling. There is another internal interface-like structure that ends within the particle exhibiting a remarkable branching, marked with a white arrow. The grain size within a distance of about $500 \mathrm{~nm}$ from the interface is between 10 and $50 \mathrm{~nm}$, the smallest grains being next to the interface. The shape of the grains is nearly equiaxed or with an elongation parallel to the particle-particle interface, and the wavy contrast inside indicates heavy strains and/or a high dislocation density in the individual grains. The inset diffraction pattern shows that the coating has a $f c c$ structure, and that the grains are randomly oriented. Figure $7 \mathrm{~b}$ shows a grain with a high density of coffee-bean-like contrasts, which are bright in this micrograph.

Figure 8 shows TEM micrographs of a 316L sample obtained by explosive compaction. Figure 8 a shows sample 1 from the outer part of the compacted cylinder, Fig. $8 \mathrm{~b}$ sample 2 from the middle, and Fig. 8c sample 3 from the inner part. The numbering of the samples is as sketched in Fig. $3 \mathrm{~b}$. Sample 1 shows a heavily deformed microstructure with an extremely high dislocation density. Sample 2 shows equiaxed grains with sizes between 10 and $50 \mathrm{~nm}$ and heavy strain contrast within the particles. A particle-particle interface is marked with an arrow. This interface seems to end within the sample, indicating the transition from loosely bonded to intimately bonded interfaces. On the lower left hand side of the interface, there seems to be a bifurcation in this interface. Sample 3 exhibits larger grains, i.e. the grain size is $0.2-0.5 \mu \mathrm{m}$, with distinct $120^{\circ}$ triple points. The larger grains have a dense population of coffee-bean-like contrasts.

\section{Discussion}

The first noticeable result for cold-sprayed $316 \mathrm{~L}$ austenitic steel coatings and explosive compacts is the fact that their structure is almost pure $f c c$, while the steel $316 \mathrm{~L}$ feedstock powder with sizes smaller than $22 \mu \mathrm{m}$ contains some amount of $b c c$ particles. In the cold-sprayed as well as the explosively compacted (d.c.) samples of this fine powder, there are virtually no $b c c$ reflections in the XRD spectra. After obtaining this result for cold-sprayed coatings, the first conceivable explanation was that $b c c$ powder particles, being less ductile than $f c c$ particles, bounce back from the substrate or, being ferromagnetic, stick to some other ferromagnetic parts in the experimental set-up, leaving a purely austenitic coating behind. The crucial experiment to verify this assumption was to perform explosive compaction of the same 316L powder: this method assures that the whole source material stays in the compacted workpiece. The X-ray spectra show that the $b c c$ phase is gone here, too, when fine powder is loaded in the direct-compaction mode. This result gives evidence that there has been a phase transformation during compaction and, most probably, during cold spraying, too.

A steel $316 \mathrm{~L}$ with a composition such as the one given in this work should be completely austenitic. Moreover, it solidifies directly into the austenitic phase. However, it is well known that ferrite is retained after welding austenitic steels of the $316 \mathrm{~L}$ type when the welding technique ensures a sufficiently large cooling rate $[22,23]$. In accordance, the formation of metastable $b c c$ structures in the case of very fine steel $316 \mathrm{~L}$ powders can be explained by the conditions for solidification in inert gas atomisation: the smallest liquid droplets are exposed to fastest heat extraction and thus highest quench rates. In consequence, the reachable undercoolings with respect to the melting point are sufficient to quench in the metastable ferrite present in the fine powders, favoured by its lower surface energy in the surrounding liquid [24]. In contrast, the solidification of bigger droplets is not triggered by such high undercoolings, and thus should result in the nucleation of the equilibrium $f c c$ structure [24, 25]. The powder particles are exposed to strong shock pressures during cold spraying or explosive compaction, leading to the transformation of metastable $b c c$ structures to austenite having a higher density. Pressure pulses of around 1 GPa [19] are obviously sufficient to overcome the activation energy of this diffusionless solid state transformation. The $f c c$ structure being the stable solid phase of stainless steel $316 \mathrm{~L}$, no further phase transformation takes place upon cooling after impact. The two methods, cold spraying and explosive compaction, compare well as to the energy input, and the degree of deformation, as well as to the deformation rates of the powder particles [14]. Therefore, it seems justified to assume that, in principle, any phase transformation that takes place during explosive compaction also takes place during cold spraying.

Figure $4 \mathrm{c}$ however shows that a slight amount of $b c c$ structures can be generated after loading a powder by a very strong shock wave. Similar to the solidification of droplets during the gas atomisation, the molten material in the jetting region of a highly loaded powder during compaction is also exposed to very high cooling rates. Here, cooling is caused by the significantly less deformed and thereby less heated surrounding material. Obviously, the cooling rates, which should be in the order of $10^{5}$ to $10^{6} \mathrm{~K} / \mathrm{s}$, are high enough to freeze in the ferritic structure, analogous to gas atomisation. This $f c c$ to bcc transformation can only be detected by XRD in samples where 10 to $20 \%$ of the material was molten after compaction, i.e. in regions close to the mandrel. Since no more than $5 \%$ of the material was in the $b c c$ structure as detected by XRD, one can assume that, upon solidification, only part of the molten material solidifies in the metastable $b c c$ structure. Since gas atomised $316 \mathrm{~L}$ powders of the fine size cut are also only partially $b c c$, and ferrite is partly retained after welding austenitic steels of the $316 \mathrm{~L}$ type when the welding technique ensures a sufficiently large cooling rate [22, 23], the estimated ferrite part in molten and resolidified $316 \mathrm{~L}$ compacts corresponds well with these findings.

As analysed by TEM, the microstructures of the explosively compacted samples compare well with the anticipated energy input along the sample radius: sample 1, taken from the outermost place of the cylindrical sample, shows high dislocation densities. In this part of the sample, the overall grain size could not be determined from TEM; it seems to be irregular. Sample 2, taken from the middle of the cylinder, exhibits a nanocrystalline microstructure with equiaxed grains, which is typically observed after dynamic recrystallisation [26]. In sample 3, taken from the centre of the cylinder, the grain size is again larger than in sample 2. The regular shape of the grains and the $120^{\circ}$ triple points indicate post-impact recrystallisation. Inside some of the recrystallised grains, as well as in the cold-sprayed sample, see Figs. $7 \mathrm{~b}$ and 8, a large amount 
of coffee-bean-like contrasts can be seen. These stem most probably from dislocation loops which are formed under impact, when moving dislocations cut each other, or dislocations climb rather than slip. During these mechanisms, numerous intrinsic and extrinsic point defects are formed, which can agglomerate to form intrinsic or extrinsic dislocation loops. In a study of cold-sprayed copper coatings, numerous dislocation loops were found [27]. In that study it was shown that (i) the generation of athermal point defects is highly probable in cold spraying and (ii) the observed density of dislocation loops can be accounted for, even if less than $1 \%$ of the generated point defects agglomerate to form loops. The observed dislocation loops in cold-sprayed copper coatings were found to be persistent even after heating the coating to $600{ }^{\circ} \mathrm{C}$. This is the case, because dislocation loops are stable even after heat treatment when they exceed a diameter of about $3 \mathrm{~nm}[27,28]$. In the light of these observations, it is not surprising to find dislocation loops in cold-sprayed and explosively compacted austenitic steel $316 \mathrm{~L}$, too.

The main difference in comparing cold-sprayed coatings of copper and steel $316 \mathrm{~L}$ is that the microstructure of $316 \mathrm{~L}$ coatings is quite uniform, while that of $\mathrm{Cu}$ coatings is very non-uniform. Cold-sprayed $316 \mathrm{~L}$ coatings show mainly nanosized equiaxed grains in the vicinity of particle-particle interfaces, see Fig. 7a, which are the product of dynamic recrystallisation. In the case of $\mathrm{Cu}$ coatings, a variety of microstructures is observed within a very small volume of, say, $1 \mu \mathrm{m}^{3}$ [8]. These different microstructures are reproduced in steel 316L samples gained by explosive powder compaction, see Fig. 8. All three shown microstructures, which in the case of steel $316 \mathrm{~L}$ are obtained by a quite different energy input during explosive compaction, can indeed be found in a single cold-sprayed $\mathrm{Cu}$ coating [8]. Frost and Ashby published deformation mechanism maps for a quantity of materials, among others $\mathrm{Cu}$ and 316 stainless steel [29]. Copper exhibits a broad transition region from one deformation mechanism to another, which 316 stainless steel does not. Though the maps are computed for rather low strains and strain rates, one can assume that copper as well as 316 stainless steel will adopt similar behaviour when subjected to high strains and strain rates. These broad transition regions of copper might be the reason why it shows such pronounced non-uniform deformation microstructures upon cold spraying. On the one hand, a physical explanation for this can be the high thermal conductivity of $\mathrm{Cu}$ : heat dissipation after impact is extremely fast, so that there is little time left to develop a microstructure closer to equilibrium. On the other hand, the temperature dependance of the modulus $\left(T_{\mathrm{M}} / \mu_{0}\right)(\mathrm{d} \mu / \mathrm{d} T)$ of stainless steel $316 \mathrm{~L}$ is much larger than for copper [29]:

$$
\frac{T_{\mathrm{M}}}{\mu_{0}} \frac{\mathrm{d} \mu}{\mathrm{d} T}(316 \mathrm{~L})=-0.85, \quad \frac{T_{\mathrm{M}}}{\mu_{0}} \frac{\mathrm{d} \mu}{\mathrm{d} T}(\mathrm{Cu})=-0.54,
$$

where $T_{\mathrm{M}}$ is the melting temperature and $\mu_{0}$ the shear modulus at room temperature. This makes the response of steel $316 \mathrm{~L}$ more sensitive to the global temperature than copper, which is reflected by the distinct microstructures corresponding to the different provenance over the radius of the cylindrical EPC steel 316L sample.

In the case of steels, one can presume that the presence of interstitial carbon may play a role in deformation. It has been reported that in austenitic steels, interstitial carbon and vacancies can form pairs that exhibit anelastic relaxation peaks in damping experiments $[30,31]$. The carbon concentration corresponds to about $7 \times 10^{25} \mathrm{C} / \mathrm{m}^{3}$ or 1 in $(2.5 \mathrm{~nm})^{3}$. The amount of vacancies generated during CS or EPC can be estimated to be in the order of $10^{-3}$ to $10^{-2}$, most of which vanish by recombination [27]. This is enough to allow the formation of a carbon-vacancy pair with every carbon interstitial. It is conceivable that these do represent an obstruction to the deformation of $316 \mathrm{~L}$. The strain rates and temperatures are far too high to lie in the vicinity of the damping peaks mentioned above, but these pairs should cause higher solution hardening than carbon interstitials alone. From former investigations it is known that $316 \mathrm{~L}$ exhibits a rather pronounced strain-rate hardening effect $[14,19]$. This is not surprising, if one takes into account that the dislocation velocity is limited by the transverse velocity of sound [32], which for steel amounts to about $3000 \mathrm{~m} / \mathrm{s}$ [33]. This means that the energy to move a dislocation diverges near the limiting velocity and has to be treated relativistically [32]. With impact velocities of 600-1200 m/s in CS and EPC, these effects should be already noticeable in the form of strain-rate hardening, since the local strain rate is strongly dependent on the impact velocity, and material velocities in jet regions can be up to two times higher than the impact velocity in the vicinity of shear instabilities [18].

Several investigations have shown that adiabatic shear instabilities and accompanying jet formation are required for successful bonding in cold spraying and explosive powder compaction $[8,18,19]$. Evidence for this jet formation can be seen in Figs. 7a and 8b. In Fig. 8b, one can see a structure looking like a bifurcation of the particle-particle interface marked with an arrow. This could most probably mark the end of a jet. The branched structures in Fig. 7a are also most probably the result of jetting. In the OM and SEM micrographs, Figs. 5 and 6, welding of the particles can be seen, where the contrast-induced interparticle interfaces seem to fade out. This is a good confirmation that successful bonding is attributed to the occurrence of adiabatic shear instabilities. Meyers and co-workers found that the formation of adiabatic shear bands leads to dynamic 'rotational' recrystallisation with the formation of approximately equi-axed nanosized grains [26], which is exactly the microstructure observed in CS steel $316 \mathrm{~L}$ coatings and sample 2 from EPC steel 316L. The fact that for higher impact energies in EPC (sample 3) post-deformation 'migrational' recrystallisation is observed is no contradiction. Here, the temperature rise was high enough to allow for further structural transformations upon cooling.

Welding is observed to a much lesser extent for the sample cut from the outside of the d.c. EPC compact than for the other samples cut from regions closer to the centre; see Figs. 5a and 8a. The particles are comparatively weakly deformed and are just above the critical conditions where bonding by shear instabilities starts to occur and the transition of the deformation pattern from a symmetric angular shape to the asymmetric parachute shape takes place $[13,14]$. This typical parachute morphology with extensive plastic flow at some regions of the interface, shown by highly elongated grains and welded areas, can be observed in the presented microstruc- 
ture. Raybould [10] connected the transition of non-bonded to bonded particles measured by the compact's strength of cohesion of steel $304 \mathrm{~L}$ powder compacts of a mean particle diameter of $100 \mu \mathrm{m}$ to an overall strain rate of $10^{7}$ to $10^{8} \mathrm{~s}^{-1}$. This corresponds exactly with the observed particle deformation at 500 to $600 \mathrm{~m} / \mathrm{s}$, presented in this paper. The impact conditions of the nitrogen sprayed cold spray coating discussed in this work were just above the critical velocity, see Fig. $6 a$ and $b$, and the impact conditions of the helium sprayed coating clearly above the critical velocity, see Fig. $6 \mathrm{c}$ and d. Here, too, welding is observed to a much lesser extent in the sample sprayed with the lower velocity, i.e. with nitrogen. This study shows that the microstructures of the investigated cold-sprayed $316 \mathrm{~L}$ coatings are quite similar to those of explosively compacted powder samples at comparable impact conditions. Large heat concentration at particle-particle interfaces by the highest impact energies in the present comparison (sample 3) results in welded interfaces and heataffected zones stretching over a width of up to $10 \mu \mathrm{m}$ inside the particles; see Fig. 6c. The generally higher heat input explains the thermally influenced microstructures shown in detail by Fig. 8c.

\section{$5 \quad$ Conclusions}

In the present investigation, the microstructures of cold-sprayed 316L stainless steel powders were investigated for cold-sprayed (CS) and explosively powder compacted (EPC) samples. By radial energy input in a cylindrical sample, shock loading in EPC can cover a wide energy range in a single experiment. The microstructures formed in regions of different energy inputs were compared to that of a CS $316 \mathrm{~L}$ coating. It was observed that the local microstructure is quite uniform in each of these regions, which is also the case for the CS coating, in contrast to copper, which in CS exhibits an extremely non-uniform microstructure. This is explained by the high thermal conductivity of copper, where heat dissipation after impact is extremely fast, so that there is little time left for an equilibrium microstructure to evolve. On the other hand, the temperature dependence of the modulus of 316 stainless steel is much larger than for copper. This makes the response of $316 \mathrm{~L}$ more sensitive to global temperature than copper, which is reflected by the distinct microstructures corresponding to the different provenance on the radius of the cylindrical EPC 316L sample. Adiabatic shear instabilities and accompanying jet formation are required for successful bonding in cold spraying and explosive powder compaction. Jet formation was observed in CS 316L samples, as well as in the EPC samples cut from regions where the energy input compares to that of CS $316 \mathrm{~L}$ coatings. This is accompanied by interparticle welding, visible in optical microscopy.

In the feedstock powders with small particle sizes, subjected to the highest cooling rates during gas atomisation, some particles were found to have a $b c c$ structure, which was not the case for larger powder sizes. This $b c c$ fraction underwent a phase transition to $f c c$ upon cold spraying and explosive compaction, which is explained by a transformation to the high-density equilibrium $f c c$ structure under shock loading. Contrary to this, large powder fractions subjected to the highest energies in EPC exhibit a small fraction of $b c c$ structure after compaction. This is explained by partial melting and re-solidification after high-energy compaction, whereby very high cooling rates were caused by significantly less deformed and thereby less heated surrounding material.

ACKNOWLEDGEMENTS The authors thank M. Papesch, Technical Centre for Weapons and Ammunition of the Federal Armed Forces for support in explosive powder compaction. This work was supported by the Deutsche Forschungsgemeinschaft by Grant No. KR 1109/3-3, which is gratefully acknowledged.

\section{REFERENCES}

1 A.P. Alkhminov, S.V. Klinkov, V.F. Kosarev, A.N. Papyrin, J. Appl. Mech. Tech. Phys. 38, 324 (1997)

2 A.P. Alkhminov, V.F. Kosarev, A.N. Papyrin, J. Appl. Mech. Tech. Phys. 39, 318 (1998)

3 A.N. Papyrin, A.P. Alkhminov, V.F. Kosarev, S.V. Klinkow, in Thermal Spray 2001: New Surfaces for a New Millennium, ed. by C.C. Berndt, K.A. Khor, E.F. Lugscheider (ASM International, Materials Park, OH, 2001), p. 423

4 J. Vlcek, H. Huber, H. Voggenreiter, A. Fischer, E. Lugscheider, H. Hallen, G. Pache, in Thermal Spray 2001: New Surfaces for a New Millennium, ed. by C.C. Berndt, K.A. Khor, E.F. Lugscheider (ASM International, Materials Park, OH, 2001), p. 417

5 T. Stoltenhoff, H. Kreye, H.J. Richter, H. Assadi, in Thermal Spray 2001: New Surfaces for a New Millennium, ed. by C.C. Berndt, K.A. Khor, E.F. Lugscheider (ASM International, Materials Park, OH, 2001), p. 409

6 T. Stoltenhoff, H. Richter, H. Kreye, J. Thermal Spray Technol. 11, 542 (2002)

7 C. Borchers, T. Stoltenhoff, F. Gärtner, H. Kreye, H. Assadi, Mater. Res. Soc. Proc. 673, 7.10 (2002)

8 C. Borchers, T. Stoltenhoff, F. Gärtner, H. Kreye, H. Assadi, J. Appl. Phys. 93, 10064 (2003)

9 R. Prümmer, Explosivverdichtung pulvriger Substanzen: Grundlagen, Verfahren, Ergebnisse (Springer, Berlin, 1987) [in German]

10 D.A. Raybould, J. Mater. Sci. 16, 589 (1981)

11 L.E. Murr, Shock Waves for Industrial Applications (Noyes, Park Ridge, NJ, 1988), p. 237

12 M.A. Meyers, L.E. Murr, J. Met. 33, 21 (1981)

13 D.G. Morris, Mater. Sci. Eng. 57, 187 (1983)

14 T. Schmidt, F. Gärtner, H. Kreye, High strain rate deformation phenomena in explosive powder compaction and cold gas spraying, in International Thermal Spray Conference, Orlando, FL, 2003, ed. by B.R. Marple, C. Moreau (ASM International, Materials Park, OH, 2003), p. 9

15 H. Kreye, Weld. J. Res. Suppl. 56, 154 (1977)

16 H. Kreye, I. Wittkamp, U. Richter, Z. Metallkd. 67, 141 (1976)

17 M. Hammerschmidt, H. Kreye, in Shock Waves and High Strain-Rate Phenomena in Metals, ed. by M.A. Meyers, L.E. Murr (Plenum, New York, 1981), p. 961

18 H. Assadi, F. Gärtner, T. Stoltenhoff, H. Kreye, Acta Mater. 51, 4379 (2003)

19 T. Schmidt, F. Gärtner, H. Assadi, H. Kreye, Acta Mater. 54, 729 (2006)

20 C. Borchers, T. Stoltenhoff, F. Gärtner, H. Kreye, J. Appl. Phys. 94, 4288 (2004)

21 T. Schmidt, F. Gärtner, H. Kreye, J. Thermal Spray Technol. 15, 488 (2006)

22 K.P. Rao, Mater. Sci. Lett. 9, 675 (1990)

23 Y.C. Lin, P.Y. Chen, Mater. Sci. Eng. A 307, 165 (2001)

24 A. Zambon, B. Badan, K. Eckler, F. Gärtner, A.F. Norman, A.L. Greer, D.M. Herlach, E. Ramous, Acta Mater. 46, 4657 (1998)

25 R.N. Wright, J.C. Bae, T.F. Kelly, J.E. Flinn, G.E. Korth, Metall. Trans. 195A, 2399 (1988)

26 M.A. Meyers, J.C. LaSalvia, V.F. Nestorenko, Y.J. Chen, B.K. Kad, in Proceedings of ReX'96: The Third International Conference on Recrystallization and Related Phenomena, ed. by T.R. McNelley (Monterey Institute of Advanced Studies, Monterey, CA, 1997), p. 279

27 C. Borchers, T. Stoltenhoff, F. Gärtner, H. Kreye, Acta Mater. 53, 2991 (2005) 
28 I. Kovacs, L. Zsoldos, Dislocations and Plastic Deformation (Pergamon, Oxford, 1973), p. 249

29 H.J. Frost, M.F. Ashby, Deformation Mechanism Maps (Pergamon, Oxford, 1982)

30 K.S.B. Rose, S.G. Glover, Acta Met. 14, 1505 (1966)
31 J.A. Slane, C. Wolverton, R. Gibala, Mater. Sci. Eng. A 370, 67 (2004)

32 P. Haasen, Physical Metallurgy (Cambridge University Press, Cambridge, 1996), p. 282

33 C.R. Nave, HyperPhysics 2006, http://hyperphysics.phy-astr.gsu.edu/ hbase/tables/soundv.html\#c3, accessed 02/23/2006 